\title{
UV ABSORPTION AND EMISSION LINES FROM HIGHLY IONIZED GAS IN THE GALACTIC HALO
}

\author{
BLAIR D. SAVAGE \\ Department of Astronomy \\ University of Wisconsin \\ Madison, Wisconsin, USA
}

\begin{abstract}
Highly ionized gas in the galactic halo has been detected through UV absorption and emission lines. In absorption the species studied include Si IV , C IV and N V. The UV emission studies have recorded C IV and $\mathrm{O}$ III]. Absorption measurements toward galactic stars reveal that the $|z|$ distribution of the gas is roughly exponential with a scale height of approximately $3 \mathrm{kpc}$ and has column densities perpendicular to the galactic plane of $\mathrm{N} \sim 2 \times 10^{13}, 1 \times 10^{14}$ and $3 \times 10^{13}$ atoms $\mathrm{cm}^{-2}$, for Si IV, C IV and NV, respectively. Similar absorption line profiles for these species suggests a common process for their origin. The presence of $\mathrm{N} \mathrm{V}$ absorption implies the existence of some gas with a temperature near $\mathrm{T} \sim 2 \times 10^{5} \mathrm{~K}$. The highly ionized absorbing gas toward distant stars in direction $b<-500$ has simple and relatively narrow line profiles (FWHM $\sim 45$ to $70 \mathrm{~km}^{-1}$ ) and small average LSR velocities while the gas in the direction $b>50^{\circ}$ reveals a complex pattern of motions with substantial inflow and outflow velocities. Galactic rotation has an appreciable effect on the absorption line profiles to very distant stars located in the low halo. C IV emission has been seen at greater than a $3 \sigma$ level of significance in 4 of 8 directions. The emission brightens toward the galactic poles and has a polar intensity I(C IV) $\sim 5000$ photons $\mathrm{cm}^{-2} \mathrm{~s}^{-1} \mathrm{ster}^{-1}$. If the emitting and absorbing gas coincide in space the measurements imply $n_{e} \sim 0.01 \mathrm{~cm}^{-3}$ and $\mathrm{P} / \mathrm{k} \sim 2000 \mathrm{~cm}^{-3} \mathrm{~K}$ for gas with $\mathrm{T} \sim$ $10^{5} \mathrm{~K}$. This phase of the gas fills only a small volume of the space ( $f \sim 0.03$ ) and accounts for only a small fraction of the total column density of gas perpendicular to the galactic plane $\left[\sim 3 \times 10^{18}\right.$ atoms $\mathrm{cm}-2$ vs $3.5 \times 10^{20}$ atoms $\mathrm{cm}-2$ for $\mathrm{H} \mathrm{I}$ and $1 \times 1020$ atoms $\mathrm{cm}-2$ for $\left.\mathrm{H}^{+}\right]$. However, the gas provides a large EUV/UV emission line flux $\left(\sim 1 \times 10^{-5} \mathrm{erg} \mathrm{cm}^{-2} \mathrm{~s}^{-1}\right)$ which corresponds to a $\mathrm{H} I$ ionizing flux of $\sim 2 \times 10^{5}$ ionizations cm-2 $\mathrm{s}^{-1}$. Gas with $\mathrm{T}$ near $2 \times 10^{5} \mathrm{~K}$ cools very rapidly. Its origin may be associated with the cooling gas of a galactic fountain flow or with thermal condensations in cosmic ray driven fountains. In the nonequilbrium cooling of a Galactic fountain, a flow rate of $4 \mathrm{MO}_{\mathrm{O}}$ / year to each side of the Galaxy is required to produce the amount of $\mathrm{N} \mathrm{V}$ absorption found in the halo while a flow rate $5 \mathrm{x}$ larger is required to produce the observed level of C IV emission.
\end{abstract}




\section{INTRODUCTION}

Highly ionized atoms in the general interstellar gas of the galactic disk were first detected through interstellar absorption line observations of $\mathrm{O}$ VI with the Copernicus satellite (Rogerson et al. 1973). Survey measurements by Jenkins (1978) of interstellar 8 VI absorption toward 72 stars demonstrated the general presence of O VI in the interstellar medium of the galactic disk. This research and parallel observational studies of the soft X-ray background (Williamson et al. 1974; McCammon et al. 1983; Marshall and Clark 1984) provided direct evidence for the existence of hot low density gas in the interstellar medium of the galactic disk.

The extension of the absorption line studies to the distant gas of the galactic halo required the launch of the International Ultraviolet Explorer (IUE) satellite in 1978. The first measures of highly ionized gas in the galactic halo were obtained with the IUE when it was used to record high resolution spectra of bright stars in the Large Magellanic Cloud (Savage and de Boer 1979). Those early spectra revealed the presence of absorption by $\mathrm{Si}$ IV and C IV in the galactic halo and have been followed by a number of surveys with IUE of highly ionized gas in the galactic disk and halo (Savage and de Boer 1981; Pettini and West 1982; Savage and Massa 1987).

The study of UV emission from highly ionized gas in the halo has progressed more slowly because of the intrinsic faintness of the emission. However, very important recent measurements were obtained by Martin and Bowyer (1990) using a low resolution nebular spectrograph flown on the space Shuttle in 1986.

In this review we discuss both absorption and emission line measurements with emphasis on the most recent work. Other recent reviews of halo gas and the hot ISM include those of Savage $(1987,1990)$, Jenkins (1987), and Spitzer (1990). The organization of this paper is as follows: The properties of the highly ionized atoms are briefly overviewed in \$2. The results from interstellar absorption line spectroscopy are discussed in $\$ 3$. Emission line studies are the subject of $\$ 4$ and several current theoretical ideas concerning the origin of highly ionized gas in the halo are discussed in $\$ 5$.

\section{PROPERTIES OF THE HIGHLY IONIZED ATOMS}

Table 1 lists information about the lithium-like resonance lines of the abundant highly ionized atoms which have transitions at wavelengths longward of the photoionization edge of $\mathrm{H}$ I at $912 \AA$. The table lists species, wavelengths, $f$ values, ionization energy required to produce $\left(\mathrm{IP}_{\mathrm{X}-1}\right)$ and to destroy $\left(\mathrm{IP}_{X}\right)$ the ions in $\mathrm{eV}$. $\mathrm{T}_{\max }(\mathrm{K})$ is the temperature at which a particular ion reaches maximum abundance assuming conditions of coronal ionization according to calculations of Shull and Van Steenberg (1982). For Si IV the effects of charge exchange are included according to the study of Baliunas and Butler (1980).

The species listed in Table 1 are ordered according to increasing energy required for their production. The production of Si IV, C IV, S VI, N V and $\mathrm{O}$ VI require approximately $34,48,73,78$ and $114 \mathrm{eV}$, respectively. With increasing energy it becomes less likely that photoionization in warm ( $T$ $\left.10^{4} \mathrm{~K}\right)$ gas is the source of ionization. The energy required to convert $\mathrm{He}^{+}$ 
TABLE 1.

Resonance lines for various highly ionized interstellar species

\begin{tabular}{lccccc}
\hline species & $\lambda(\AA)$ & $\mathrm{f}$ & $\mathrm{P}_{\mathbf{X}-1}$ & $\mathbb{P}_{\mathbf{X}}$ & $\mathrm{T}_{\max }(\mathrm{K})$ \\
\hline Si IV & 1402.77 & 0.262 & 33.5 & 45.1 & $0.6 \times 10^{5}$ \\
Si IV & 1393.76 & 0.528 & & & \\
C IV & 1550.76 & 0.097 & 47.9 & 64.5 & $1.0 \times 10^{5}$ \\
C IV & 1548.19 & 0.194 & & & \\
S VI & 944.52 & 0.210 & 72.5 & 88.0 & $2.0 \times 10^{5}$ \\
S VI & 933.38 & 0.426 & & & \\
N V & 1242.80 & 0.0757 & 77.5 & 97.9 & $1.8 \times 10^{5}$ \\
N V & 1238.81 & 0.152 & & & \\
O VI & 1037.63 & 0.0648 & 113.9 & 138.1 & $3.0 \times 10^{5}$ \\
O VI & 1031.95 & 0.130 & & & \\
\hline
\end{tabular}

to $\mathrm{He}^{+2}(54 \mathrm{eV})$ is of particular importance in determining which species might be created by photoionization in warm gas versus electron collisional ionization in hot gas. Most hot stars containing helium have strong discontinunities at $54 \mathrm{eV}$, and are unlikely to be strong sources of radiation more energetic than $54 \mathrm{eV}$. Therefore, among the species listed in Table 1, $\mathrm{S}$ VI, N V and O VI stand out as the best for diagnostic information on the hot phase of the interstellar medium. These ions, if created under conditions of equilibrium collisional ionization, will probe gas in the temperature range from about $1 \times 10^{5}$ to $4 \times 10^{5} \mathrm{~K}$. Unfortunately, the resonance lines of both S VI and O VI occur at wavelengths that are shortward of the $1150 \AA$ limit of IUE and the spectrographs aboard the Hubble Space Telescope. Therefore, data on these important ions in halo gas will need to wait for future satellites such as Lyman/FUSE. In the wavelength region for which halo gas has been probed in absorption by IUE $(\lambda>1150 \AA)$, the most important hot gas diagnostic ion is N V.

Hot collisionally ionized gas will produce a UV emission line spectrum that will include most of the resonance lines listed in Table 1. In addition the gas will produce forbidden emission lines and lines due to transitions between excited electronic states. As an illustration of what might be expected, Table 2 provides a list of the line flux for the strongest lines produced by a cooling conductive interface with solar abundances during two times in its evolution. The photon fluxes are from Borkowsky, Balbus, and Fristrom(1990) and were calculated by following the full nonequilibrium time evolution for a medium evolving from $7.5 \times 10^{5} \mathrm{~K}$ with an initial number density of $n\left(\mathrm{H}^{+}\right)=2.3 \times 10^{-3} \mathrm{~cm}^{-3}$. The strongest UV lines produced by the interface are C III $\lambda 977$, C III] $\lambda 1909$, C IV $\lambda \lambda 1550,1551$ and O VI $\lambda \lambda 1032,1038$. 
TABLE 2.

UV emission line fluxes produced during the nonequilibrium cooling of gas in a conductive interface ${ }^{a}$

\begin{tabular}{lllc}
\hline species & $\lambda(\AA)$ & $\begin{array}{r}\text { (photons } \mathrm{cm}^{-2} \mathrm{~s}^{-1} \text { ) } \\
\mathrm{t}=2.8 \times 10^{5} \mathrm{yr}\end{array}$ & $\begin{array}{c}\text { (photons } \mathrm{cm}^{-2} \mathrm{~s}^{-1} \text { ) } \\
\mathrm{t}=2.2 \times 10^{6} \mathrm{yr}\end{array}$ \\
\hline He II & 1640 & 101 & 13 \\
C II & 1335 & 143 & 255 \\
C II] & $2324-2328$ & 237 & 478 \\
C III & 977 & 995 & 1107 \\
C III] & 1909 & 414 & 680 \\
C IV & 1548,1551 & 1110 & 525 \\
N II] & 2140,2142 & 39 & 71 \\
N III & 990 & 31 & 64 \\
N IV] & 1486 & 35 & 53 \\
N V & 1239,1243 & 174 & 106 \\
O III] & 1661,1667 & 46 & 123 \\
O IV] & $1402-1413$ & 95 & 226 \\
O V] & 1218 & 183 & 269 \\
O VI & 1032,1038 & 606 & 684 \\
Si III & 1207 & 139 & 139 \\
Si III ] & 1892 & 118 & 182 \\
Si IV & 1394,1403 & 104 & 100 \\
\hline
\end{tabular}

a. From the nonequilibrium calculations of Borkowsky, Balbus, and Fristrom (1990).

\section{UV ABSORPTION LINE STUDIES}

Most of the UV absorption line observations of halo gas have been obtained with the short wavelength spectrograph aboard the IUE satellite which is capable of recording absorption produced by Si IV, C IV and N V in addition to the absorption arising in atoms found in moderately ionized and neutral gas (e.g. H I, O I, C II, Fe II, Si II, Mg II, S II, Si III, Al III, etc.). The earliest IUE measurements generally consisted of spectra based on single exposures and typically had rather low signal to noise ratios. In contrast for some of the most recent spectra, which are based on averaging four or more images, the data quality is quite good. The higher signal to noise is crucial for the detection of absorption by $\mathrm{N}$ V which is the best hot gas diagnostic in the IUE wavelength range. The principal results relating to IUE observations of highly ionized gas in the halo are found in: Savage and de Boer $(1979,1981)$, Pettini and West (1982), Fitzpatrick and Savage (1983), Savage and Massa (1987), and Savage, Massa and Sembach (1990). A summary of results follows:

Absorption by $\mathrm{Si}$ IV, C IV , N V , and O VI is found in the general interstellar medium of the galactic disk away from pronounced photoionized $\mathrm{H}$ II regions. The gas is patchy and exhibits a spread in the average line of 
sight density, $n$ (ion) $\left[\mathrm{cm}^{-3}\right]$, of about $\pm 3 x$. The average midplane density of this gas is listed in Table 3. Measures of the distribution of the gas away from the galactic plane have been inferred from studies of the shape of curves of $\mathrm{N}$ (ion) $\mid$ sinb $\mid$ versus $|z|$ for measurements toward large numbers of stars in the disk and halo. The data are consistent with an exponential distribution of density with scale height, $\mathrm{h} \sim 3 \mathrm{kpc}$ for Si IV and C IV and $\sim 2 \mathrm{kpc}$ for N V (Savage and Massa 1987). A similar estimate for O VI does not exist because the sample of Jenkins (1978) only included 3 stars with $|z|>1 \mathrm{kpc}$. These scale heights are substantially larger than the $0.5 \mathrm{kpc}$ scale height for the extended component of H I (see Lockman 1984 and this volume) or for the 1 kpc scale height for the electrons (see Reynolds 1989 and this volume).

The total observed column densities for the high ions through the halo on one side of the galaxy, $N_{\infty}|\operatorname{sinb}|$, are listed in Table 3 . Note that for $N \mathrm{~V}$, $\mathrm{N}_{\infty}|\operatorname{sinb}| \sim 3 \times 10^{13}$ atoms $\mathrm{cm}^{-2}$. If the $\mathrm{N} \mathrm{V}$ were produced by equilibrium collisional ionization balanced by radiative recombination in gas having solar abundances near $2 \times 10^{5} \mathrm{~K}$, the amount of $\mathrm{N} \mathrm{V}$ implies the existence of $\sim 3 \times 10^{18}$ atoms $\mathrm{cm}^{-2}$ of hot gas. This number should be compared to the column densities of $\mathrm{H}_{\mathrm{I}}$ and electrons perpendicular to the galactic plane $\left[\mathrm{N}_{\infty}(\mathrm{HI})|\operatorname{sinb}| \sim 3.5 \times 10^{20}\right.$ atoms $\mathrm{cm}^{-2}$ (Lockman, Hobbs and Shull 1986) and $\mathrm{N}_{\infty}(\mathrm{e})|\operatorname{sinb}| \sim 1 \times 10^{20}$ atoms $\mathrm{cm}^{-2}$ (Reynolds, this volume) ]. Although the $2 \times 10^{5} \mathrm{~K}$ gas represents about only $1 \%$ of the total mass column density, it provides important information about those galactic processes that cause gas to be found a such large distances from the galactic plane.

\section{TABLE 3.}

Absorption line column densities and midplane densities for highly ionized halo gas.

\begin{tabular}{ccccc}
\hline Ion & $\begin{array}{c}\text { midplane } \\
\text { density }\end{array}$ & $\begin{array}{c}\text { Observations } \\
\text { of Halo Stars }\end{array}$ & $\begin{array}{c}\text { Photoionized } \\
\text { Halo }\end{array}$ & $\begin{array}{c}\text { Cooling } \\
\text { Fountainc }\end{array}$ \\
\hline $\mathrm{n}_{\mathrm{o}}\left(\mathrm{cm}^{-3}\right)$ & $\left\langle\mathrm{N}_{\infty}|\operatorname{sinb}|>\right.$ & $\mathrm{N}_{\infty}|\operatorname{sinb}|$ & $\mathrm{N}_{\infty}|\operatorname{sinb}|$ \\
\hline Si IV & $2 \times 10^{-9}$ & $\sim 2 \times 10^{13}$ & $1.3 \times 10^{14}$ & $(3.3-6.4) \times 10^{12}$ \\
C IV & $7 \times 10^{-9}$ & $\sim 1 \times 10^{14}$ & $1.2 \times 10^{14}$ & $(4.3-7.9) \times 10^{13}$ \\
N V & $3 \times 10^{-9}$ & $\sim 3 \times 10^{13}$ & $7.3 \times 10^{11}$ & $(2.8-3.6) \times 10^{13}$ \\
O VI & $2 \times 10^{-8}$ & $>3 \times 10^{13}$ & $1.4 \times 10^{11}$ & $(5.8-6.0) \times 10^{14}$
\end{tabular}

a. Observations are from Savage and Massa (1987) for Si IV , C IV and N V and from Jenkins (1978) for O VI.

b. Photoionized halo model of Hartquist,Pettini and Tallant (1984). Assumes, $\mathrm{n}_{\mathrm{o}}\left(\mathrm{H}^{+}\right)=0.003$ atoms $\mathrm{cm}^{-3}$, a gas scale height, $\mathrm{h}=3 \mathrm{kpc}$ and an estimate of the density of ionizing Lyman continuum photons from the QSO background radiation impinging on the outer region of the halo of $n(\gamma)=$ $1 \times 10^{-6} \mathrm{~cm}^{-3}$.

c. Nonequilibrium cooling fountain calculation of Edgar and Chevalier (1986). Assumes a mass flow rate of $4 \mathrm{M} \odot /$ year to each side of the galactic plane. This flow produces a C IV emission intensity of I(C IV $)=890$

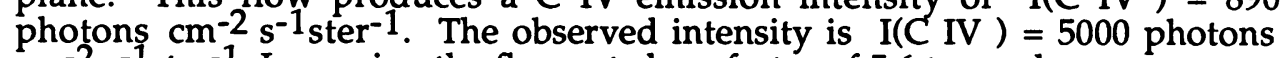
$\mathrm{cm}^{-2} \mathrm{~s}^{-1}$ ster $^{-1}$. Increasing the flow rate by a factor of 5.6 to produce agreement with the emission line data would result in a $5 x$ overproduction of $\mathrm{N} V$. 
The evidence for a jump or substantial increase in $n$ (Si IV) or $n$ (C IV) near $|z| \sim 1 \mathrm{kpc}$ as proposed by Pettini and West (1982) and hinted at in the data of Savage and Massa (1987) is not strong. Such a jump is predicted by models involving the origin of $\mathrm{Si}$ IV and C IV through photoionization by extragalactic radiation. However, the existence of such a jump is inconsistent with the galactic rotational analysis of profiles to very distant stars at low latitudes $\left(|\mathrm{b}|<15^{\circ}\right.$ ) with $|\mathrm{z}| \sim 1$ to $3 \mathrm{kpc}$ (Savage, Massa and Sembach 1990). Jumps are difficut to see in plots of $N($ ion $)|\operatorname{sinb}|$ versus $|z|$ because of the degree of variability or patchyness of the absorption. Local galactic structure may also influence our view. The column densities of Pettini and West (1982) mostly refer to stars near the sun while the more recent measures include many stars at very large distances from the sun (up to $11 \mathrm{kpc}$ ) and thus involve substantial averaging over the galaxy. More work is needed to better define the true $|z|$ distribution of the highly ionized atoms.

In those cases where absorption line profiles for the highly ionized species have been well measured by obtaining multiple IUE spectra, the profiles for Si IV, C IV and NV are quite similar in shape and very different from profiles for the low ions or for species directly produced by photoionization such as Al III (see Savage et al 1989; Savage, Massa and Sembach 1990). This result suggests that $\mathrm{Si}$ IV, C IV and N V may be created in the same regions of interstellar space by a similar process.

The kinematical information provided by the high ionization lines is quite interesting. However, the interpretation of the results have been hampered by the general patchyness of the absorption, by the relatively low signal to noise ratios of individual IUE spectra, and by the complexity of the kinematics. In a sample of stars situated in the general direction of the south galactic pole, ( $b<-50^{\circ}$ ), Danly (1987) found the high ionization line profile structure for stars at $|\mathrm{z}| \sim 1$ to $2 \mathrm{kpc}$ to be relatively simple. For example, the lines of Si IV and C IV typically have widths (FWHM) of about 45 to $70 \mathrm{~km} \mathrm{~s}^{-1}$ and average LSR velocities $\langle\mathrm{v}\rangle \sim-10$ to $+20 \mathrm{~km} \mathrm{~s}^{-1}$. The view in this direction suggests a relatively quiescent region of space. The exact opposite behavior is found when looking at distant stars toward the north galactic pole, $\left(b>+50^{\circ}\right)$, where the profiles are complex, involving positive and negative velocities and multiple components with a preference for negative velocities. In this direction the profile widths (FWHM) vary from about 45 to $100 \mathrm{~km} \mathrm{~s}^{-1}$. The direction of the north galactic pole suggests a disturbed region with substantial downflow. Clearly, local galactic structure is greatly influencing our view of the $\mathrm{z}$ motions of the gas.

The effect of differential galactic rotation on the appearance of interstellar absorption lines of the highly ionized gas in the halo was first studied by Savage and de Boer $(1979,1981)$ and by Savage and Massa (1987). The data from the 1987 study of 40 stars suggested that substantial deviations from corotation occur in highly ionized halo gas and that rotation may cease completely at about $|\mathrm{z}| \sim 3 \mathrm{kpc}$. However, much additional work will be needed to confirm that result. In a new observing program Savage, Massa and Sembach (1990) are obtaining high quality IUE line profiles for selected halo stars for which galactic rotation effects are expected to be large. In a detailed analysis of the profiles toward HD 163522, a B1 Ib halo star at a line of sight distance of $9 \mathrm{kpc}$ and a $\mathrm{z}$ distance of $-1.5 \mathrm{kpc}$ in the direction $\mathrm{l}=350^{\circ}$ and $b=-90$, it was found that the profiles of interstellar species known to have large scale heights (e.g. Si IV, C IV and N V) are significantly more affected by galactic rotation than the profiles of species having smaller scale 
heights (e.g. H I, Fe II, Mg II, S II, etc). Simple model calculations were performed to understand this result. In the case of gas with a small scale height, the sight line simply runs out of gas before the effects of galactic rotation become appreciable. Such studies demonstrate that once the galactic rotation curve is known for matter away from the galactic plane, it will be possible to infer from the observed line profiles the actual run of density with distance away from the galactic plane for a large number of interstellar species.

\section{UV EMISSION LINE STUDIES}

The study of UV emission lines from highly ionized atoms in halo gas was significantly advanced with the flight of the Berkeley EUV/FUV Nebular Grating Spectrophotometer on the space shuttle in January 1986 (Martin and Bowyer 1990). The $0.1 \times 4.0$ degree field of view of the spectrophotometer recorded spectra of the UV background in eight directions with $13 \AA$ resolution from 1350 to $1900 \AA$. Emission from C IV $\lambda 1550 \AA$ at a greater than $3 \sigma$ level was found for 4 of 8 directions while $O$ III] $\lambda$ 1663,1667 emission $(3 \sigma)$ was found in 2 of 8 directions. In addition, the summed high latitude spectrum reveals the $3 \sigma$ detection of the O IV/Si IV $\lambda 1401$ and N III] $\lambda 1750$ emission lines. The data are limited but suggest several trends. The $C$ IV emission appears to be galactic pole brightened and anti-correlated with the column density of neutral hydrogen. The intensity of C IV emission toward the galactic polar directions is, I(C IV) $\sim 5000$ photons $\mathrm{cm}^{-2} \mathrm{~s}^{-1} \mathrm{ster}^{-1}$. Martin and Bowyer (1990) demonstrated that the emission is not likely due to detector fixed pattern noise, earth atmospheric airglow, zodiacal light, cool star chromospheres, or hot stars. They proposed that the emission is probably from collisionally ionized interstellar gas with $\mathrm{T}$ near $10^{5} \mathrm{~K}$ and suggested that the gas is likely distant halo gas. A possible complication is that some of the emission may be associated with the negative velocity complex that extends over much of the north galactic pole (Wesselius and Fejes 1973; Danly 1989). In this case the emission process might be radiative shocks produced when the clouds strike the $\mathrm{H}$ I disk. To produce the observed features a shock velocity of about $100 \mathrm{~km} \mathrm{~s}^{-1}$ is required, while the complex has radial velocities of about 50 to $70 \mathrm{~km} \mathrm{~s}^{-1}$. However, projection effects may play a role and the actual velocities may be substantially larger than the observed radial velocities. An inspection of Bell Lab $21 \mathrm{~cm}$ data reveals that strong $H$ I emission between -40 and $-70 \mathrm{~km} \mathrm{~s}^{-1}$ is seen for all the north galactic polar pointings of the Berkeley spectrophotometer. The data for the one southern pointing (near $\mathrm{l}=216^{\circ}$ and $\mathrm{b}=-390$ ) is in a direction for which the $21 \mathrm{~cm}$ emission is restricted to the velocity range from about -40 to $+40 \mathrm{~km} \mathrm{~s}^{-1}$. For this direction C IV emission is possibly detected with an intensity of $2700(+1000,-1500)$ photons $\mathrm{cm}^{-2} \mathrm{~s}^{-1}$ ster $^{-1}$. It is important to obtain additional emission measurements for other directions.

If the observed emission is due to collisional excitation of C IV and OIII] in gas under conditions of equilibrium collisional ionization near $10^{5} \mathrm{~K}$, the required emission measure of $10^{5} \mathrm{~K}$ gas is $\sim 0.01 \mathrm{~cm}^{-6} \mathrm{pc}$, assuming cosmic abundances. The observed emission has important implications for the properties of the highly ionized gas found at large distances from the galactic plane. In collisionally excited gas the C IV emission line intensity, $\mathrm{I}(\mathrm{C} \mathrm{IV}) \propto \int \gamma(\mathrm{T}) \mathrm{n}(\mathrm{C}$ IV $) \mathrm{n}_{\mathrm{e}} \mathrm{dx}$, where $\gamma(\mathrm{T})$ is the electron collision excitation 
rate coefficient. Absorption data gives the C IV column density, $\mathrm{N}(C$ IV $)=\int \mathrm{n}(\mathrm{C}$ IV) $\mathrm{dx}$, integrated out to the distance of the background star. If the emitting and absorbing gas are assumed to coincide in space, it is possible to estimate the electron density in the region containing $C$ IV. Assuming $\mathrm{T} \sim 10^{5} \mathrm{~K}$ and using $\mathrm{I}(\mathrm{C} \mathrm{IV})=5000$ photons $\mathrm{cm}^{-2} \mathrm{~s}^{-1}$ ster $^{-1}$ and $\mathrm{N}_{\infty}$ (C IV) $|\operatorname{sinb}|=1 \times 10^{14}$ atoms $\mathrm{cm}^{-2}$, the result is $\mathrm{n}_{e} \sim 0.01 \mathrm{~cm}^{-3}$. This implies a thermal pressure $\mathrm{P} / \mathrm{K}=2 \mathrm{nT} \sim 2000 \mathrm{~cm}^{-3} \mathrm{~K}$. Both the electron density and the pressure increase if the assumed gas temperature is increased. The filling factor, $f$, of the highly ionized gas can also be obtained if the path length occupied by the emitting and absorbing region is known. Taking the path length to be the exponential scale height estimated from the absorption line data $(3 \mathrm{kpc})$ and assuming equilibrium ionization we obtain $\mathrm{f}=0.01$ for $\mathrm{T} \sim 10^{5} \mathrm{~K}$. The filling factor increases to 0.06 for the nonequilibrium ionization calculations performed by Martin and Bowyer(1990). In either case the gas phase sampled in the CIV absorption and emission lines seems to occupy only a small fraction of the sight line. The cooling time for gas with the conditions estimated above is short (about $3 \times 10^{5} \mathrm{yrs}$.). The assumption of collisional ionization equilibrium is only valid if the cooling time is much longer than the time required to establish ionization equilibrium which is about $5 \times 10^{6}$ yrs. Clearly nonequilibrium ionization effects must be considered (see \$5).

The C IV emission results would seem to have important implications for the energy budget of the ISM if they are characteristic of the galaxy as a whole. Scaling from the C IV data, the implied emission line flux integrated over the entire spectrum is approximately $1 \times 10^{-5} \mathrm{erg} \mathrm{cm}^{-2} \mathrm{~s}^{-1}$ for $\mathrm{T} \sim 1 \times 10^{5} \mathrm{~K}$. If this flux is typical of the entire galaxy, it implies a luminosity of $\mathrm{L} \sim 4 \times 10^{40}$ $\mathrm{erg} \mathrm{s}^{-1}$ or $\sim 13 \%$ of the estimated injection power of supernovae (Martin and Bowyer 1990). In addition, the $\mathrm{HI}$ ionizing flux from this radiation is estimated to be $2 \times 10^{5}$ ionizations $\mathrm{cm}^{-2} \mathrm{~s}^{-1}$ which is about 20 times larger than the ionization rate estimated by Fransson and Chevalier (1985) to be associated with the QSO EUV background and about 20x smaller than the rate required to produce the diffuse H $\alpha$ background (Reynolds 1984).

\section{ORIGIN OF THE HIGHLY IONIZED ATOMS}

In galactic fountain models, gas is found in the halo because of dynamic phenomena which result in the ejection of gas from the disk. In magnetic and cosmic ray supported halo models, the pressure of cosmic rays interacting with the galactic magnetic field is employed to support the gas found at large distances from the galactic plane. In the following, we examine how these two classes of theories are able to explain some of the existing observations of highly ionized gas in the halo.

\subsection{Galactic Fountain Models}

The term "galactic fountain" describes a process in which hot gas rises above the galactic plane before cooling and condensing to form clouds which then fall to the plane (Shapiro and Field 1976). The height to which hot gas will rise and the expected velocities of the condensations in the cooling gas depend on the temperature of the gas at the base of the fountain, the rate of cooling of the upflowing gas, and whether or not there are heating processes 
occurring at large $z$. Estimates of the expected velocities show that fountains driven by hot gas $\left(1\right.$ to $\left.2 \times 10^{6} \mathrm{~K}\right)$ can roughly reproduce the pattern of motions observed in the high velocity cloud phenomena seen in the neutral hydrogen $21 \mathrm{~cm}$ line (see Bregman 1980) while fountains driven by cooler gas $\left(2\right.$ to $3 \times 10^{5} \mathrm{~K}$ ) have velocities which are more compatible with the existing optical and ultraviolet data for gas in the low halo (Houck and Bregman 1990).

The filling factor of the hot gas in the galactic disk is currently very uncertain. If the filling factor is large, the flow of hot bouyant gas into the halo may occur quite freely. Gas with a temperature of $10^{6} \mathrm{~K}$ has a thermal scale height of $6 \mathrm{kpc}$ in the galactic gravitational field and it will attempt to assume a distribution in $|z|$ compatible with that scale height. If the filling factor of hot gas in the galactic disk is small, individual bubbles of hot gas may experience difficulty in pushing the cooler matter away. With the realization that the cooler gas of the Milky Way also has an extended component with a scale height of $\sim 0.5 \mathrm{kpc}$ for the neutral phase (Lockman, this volume ) and $\sim 1 \mathrm{kpc}$ for the ionized phase (Reynolds this volume), it has become clear that the flow of hot gas into the halo may not occur as freely as previously imagined (Cox, this volume and references therein). Recent models for the flow of gas into the halo therefore have generally considered the phenomena occurring in regions of multiple supernovae which create superbubbles of hot gas that may have a chance of breaking through the cooler matter of the galactic disk (e.g. see MacLow and McCray 1988 ; Norman and Ikeuchi 1989). In this new type of model referred to by Norman and Ikeuchi (1989) as "chimney model," it is proposed that the connection between gas in the disk and halo is through "chimneys" which are the consequence of superbubbles bursting out of the galactic disk, forming collimated structures through which hot gas flows into the halo. Cox (this volume) has argued, however, that even this chimney model is likely to be suppressed by the high cosmic ray and magnetic pressures which are generally believed to be present in the halo. Models by Tomisaka (this volume) have tended to confirm this expectation but Cesarsky (also this volume) points out that the containment may be subject to instabilities.

The required circulation rate of gas from the disk into the halo and back can be estimated from the measurements of $\mathrm{N} \mathrm{V}$ absorption and $\mathrm{C}$ IV emission. Cooling gas of a galactic fountain can explain the fUE observations of $\mathrm{N} \mathrm{V}$ absorption (see Table 3), provided the fountain flow rate is about $6 \times 10^{-9} \mathrm{M}_{\odot} \mathrm{yr}^{-1} \mathrm{pc}^{-2}$ (Edgar and Chevalier 1986). This corresponds to a galactic flow rate of $4 \mathrm{M}_{\odot \mathrm{yr}^{-1}}$ to each side of the galactic plane. A flow rate 5 times larger is required to explain the C IV emission observations of Martin and Bowyer (1990). A difference this large appears to point toward a definite problem in the way the measurements have been interrelated or in the basic assumptions of the cooling fountain calculations. Perhaps $100 \mathrm{~km} \mathrm{~s}^{-1}$ shocks are enhancing the C IV emission over the north galactic polar region as discussed in \$4. Another possiblility is that the inclusion of more details in the cooling gas models will modify the various line ratios. The work of Benjamin and Shapiro presented at this meeting suggests that more realistic nonequilibrium cooling models including the effects of photoionization provide better representations of the measurements. 
Another explanation for the support of gas at large distances away from the galactic plane is found in those models which involve pressure support from the galactic magnetic field and cosmic rays. Such models, have been proposed by Hartquist, Pettini and Tallant (1984), Chevalier and Fransson (1984), Hartquist and Morfill (1986) and Bloemen (1987). In one model the $B$ field is parallel to the galactic plane and the support is via magnetic pressure which is affected by the cosmic ray pressure (see Bloemen 1987). In the other models the B field is perpendicular to the galactic plane and the pressure support is from the streaming motions of cosmic rays along the $B$ field (see Hartquist and Morfill 1986).

In some of the models, it is proposed that the ionization of the gas, and in particular the production of the highly ionized species, is by photoionization from radiation produced by hot galactic stars (Bregman and Harringtion 1986) or from the extragalactic EUV background (York 1982; Hartquist, Pettini and Tallant 1984; Fransson and Chevalier 1985). Photoionized halo models have been successful at providing a possible explanation for the observed amounts of Si IV and C IV in gas at large distances from the galactic plane (see Table 3). However, the photoionization models have not been successful in explaining the observed amount of $\mathrm{N} \mathrm{V}$ which appears to require the existence of collisionally ionized gas near $200,000 \mathrm{~K}$.

\subsection{Composite Models}

In a recent extension of the photoionization models by Ito and Ikeuchi (1988), it was found necessary to include three gas phases: 1) A neutral gas phase with $\mathrm{T}<10^{4} \mathrm{~K}$. 2) A photoionized gas phase containing C IV and Si IV with $\mathrm{T}$ near $10^{4} \mathrm{~K} .3$ ) A hot collisionally ionized gas phase with $\mathrm{T} \sim 2 \times 10^{5} \mathrm{~K}$ to explain the existence of $\mathrm{N} \mathrm{V}$. The support of gas in this model was proposed to be a galactic fountain driven by superbubble phenomena. However, another possibility is that the gas is supported by diffusing cosmic rays and that the high temperatures required to produce $\mathrm{N} V$ arise from cosmic ray heating of the gas (Hartquist and Morfill 1986). If the processes producing Si IV, C IV and N V were as different as those proposed in such models it is difficult to understand why these three ions have such similar absorption line profile shapes.

\section{ACKNOWLEDGEMENTS}

Helpful comments about draft versions of this manuscript were provided by Donald Cox and Kenneth Sembach. The author acknowledges support for research on galactic halo gas from NASA grant NAG-186. 


\section{REFERENCES}

Baliunas, S.L., and Butler, S.E. (1980) Ap.J. (Letters ), 235,L45.

Bloemen, J.B.G.M. (1987) Ap.J., 322,694.

Borkowsky,K.J., Balbus, S.A., and Fristrom, C.C. (1990) Ap.J., 355, 501.

Bregman,J.N. (1980) Ap.J., 236,577.

Bregman, J.N., and Harrington, P.J. (1986) Ap.J., 309,833.

Chevalier, R. A., and Fransson, C. (1984) Ap.J. (Letters ), 279,L43.

Danly, L. (1987) Ph.D. Thesis, University of Wisconsin-Madison. . (1989) Ap.J., 342, 785.

Edgar, R.J., and Chevalier, R.A. (1986) Ap.J. (Letters), 310, L27.

Fitzpatrick, E.L., and Savage, B.D. (1983) Ap.J., 267,93.

Fransson, C., and Chevalier, R.A. (1985) Ap.J., 296, 35.

Hartquist, T.W., and Morfill, G.E. (1986) Ap.J., 311, 518.

Hartquist, T.W., Pettini, M., and Tallant, A. (1984) Ap.J., 276, 519.

Houck, J.C., and Bregman, J.N. (1990) Ap.J., 352, 506.

Ito,M., and Ikeuchi, S. (1988) Publ. Astron. Soc. Japan, 40, 403.

Jenkins, E. B. (1978) Ap.J. , 220, 107.

(1987) in Exploring the Universe with the IUE Satellite,

(Dordrecht:D.Reidel.Pub.Co.),p.531.

Lockman, F. J. (1984) Ap.J., 283, 90.

Lockman, F.J., Hobbs, L.M., and Shull,M. (1986) Ap.J. , 301, 380.

Mac Low,M.-M., and McCray , R.C. (1988) Ap.J., 324,776.

Martin, C., and Bowyer, S. (1990) Ap.J., 350, 242.

Marshall,F.J., and Clark,G.W.(1984) Ap.J. , 287, 633.

McCammon, D., Burrows, D.N., Sanders, W.T., and Kraushaar, W.L. (1983) Ap.J., 269, 107.

Norman, C.A., and Ikeuchi,S. (1989) Ap.J. , 345, 372.

Pettini, M., and West, K.A. (1982) Ap.J., $260,561$.

Reynolds, R.J. (1989) Ap.J.(Letters), 339, L29. .(1984) Ap.J., 282, 191.

Rogerson, J. B., York, D.G., Drake, J.F., Jenkins, E.B., Morton, D.C., and Spitzer, L. (1973) Ap.J. (Letters ), 279,L43.

Savage, B.D. (1987) in Interstellar Processes, eds. D..Hollenbach and H.A.Thronson,Jr., (Dordrecht:D.Reidel Pub.Co.), p.123 .

Savage, B.D. (1990) in Evolution of the Interstellar Medium , ed, L. Blitz, (PASP Conference Proceedings), in press.

Savage, B.D., and deBoer, K.S. (1979) Ap.J. ( Letters) , 230, L77. (1981) Ap.J. , 243, 460.

Savage, B.D.JJenkins, E.B., Joseph, C. L., and de Boer, K.S. (1989) Ap.J. , 345, 393.

Savage, B.D., and Massa, D. (1987) Ap.J., 314, 380.

Savage, B.D., Massa, D., and Sembach, K. (1990) Ap.J. , 355 ,114.

Shapiro, P.R., and Field, G.B. (1976) Ap.J. , 205, 762.

Shull, M.J, and van Steenberg, M. (1982) Ap.J. (Supplment), 48, 95.

Spitzer, L. (1990) Ann. Rev. Astr. Ap., (in press).

Wesselius, P.R., and Fejes,I. (1973) Astr.Ap., 24,15.

Williamson, F.O., Sanders, W.T., Kraushaar, W.L., McCammon, D., Borken,R., and Bunner, A.N. (1974) Ap.J.(Letters), 193, L133.

York, D.G. (1982) Ann. Rev. Astr. Ap., 20, 221. 


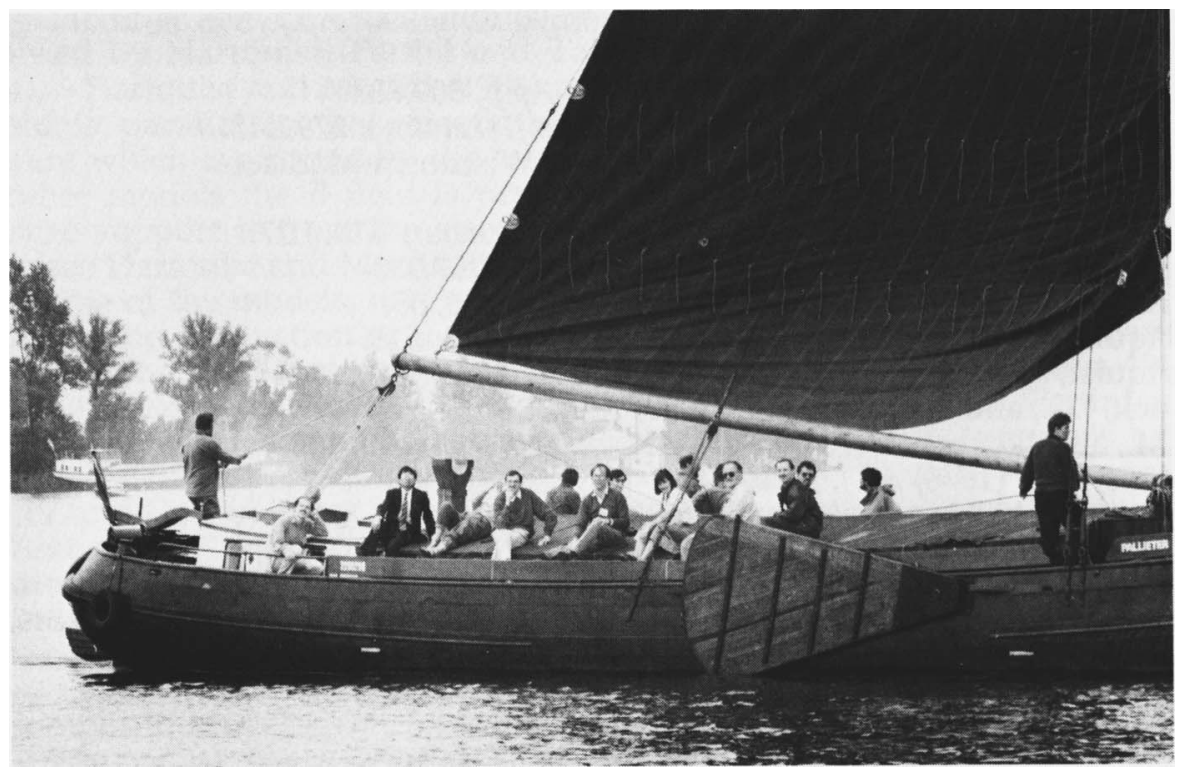

